\title{
Effects of Virtual Reality Based Therapeutic Exercise on the Upper Extremity Function and Activities of Daily Living in Patients with Acute Stroke: A Pilot Randomized Controlled Trial
}

\author{
Hwi-young Cho ${ }^{1}$, Eseul Song ${ }^{2}$, Jong-Hoon Moon $^{3}$, Suk-Chan Hahm ${ }^{4}$ \\ ${ }^{1}$ Associate Professor, Department of Physical Therapy, Gachon University, Incheon, Republic of Korea, ${ }^{2} P h D$ \\ Student, Department of Health Policy and Hospital Management, Graduate School of Public Health, Korea \\ University, Seoul, Republic of Korea, ${ }^{3}$ Assistant Professor, Department of Occupational Therapy, Kyungdong \\ University, Wonju, Republic of Korea, ${ }^{4}$ Assistant Professor, Graduate School of Integrative Medicine, CHA \\ University, Seongnam, Republic of Korea
}

\begin{abstract}
Background: Virtual reality-based therapeutic exercise (VRTE) has been considered as a rehabilitative intervention for neurological deficits. The aim of this study was to compare the effects of VRTE and occupational therapy (OT) on the upper extremity function and activities of daily living in patients with acute stroke.
\end{abstract}

Method: Twenty-four patients who had suffered a stroke within the last 30 days before enrollment participated in this study. They were randomly assigned to either the VRTE group $(n=12)$ or the OT group $(\mathrm{n}=12)$. Twenty sessions were conducted in all. To quantify the upper extremity function, the Jebsen-Taylor hand function test (JT) and Fugl-Meyer Assessment (FMA) of the upper limb were used, and the grip strength (GS) was assessed. To assess activities of daily living, the modified Barthel index (MBI) was used.

Results: In the VRTE group, JT ( $p=0.002)$, FMA $(p=0.002)$, GS $(p=0.002)$, and MBI $(p=0.002)$ showed a significant improvement after the intervention. In the OT group, JT ( $p=0.002)$, FMA ( $p=0.001)$, GS (p $=0.002)$, and MBI $(\mathrm{p}=0.001)$ significantly improved after the intervention. However, compared with OT, VRTE did not show significant improvements in upper extremity function and activities of daily living.

Conclusions: This study suggests that the early approach of VRTE is not superior to OT for the improvement of upper limb function and activities of daily living in patients of stroke.

Keywords: Activities of daily living, occupational therapy, upper limb function, acute stroke, therapeutic exercise, virtual reality.

\section{Introduction}

Neurological deficits are common after stroke

\section{Corresponding Author:}

Suk-Chan Hahm, PhD, PT

Graduate School of Integrative Medicine, CHA

University, CHA Bio Complex, 355, Pangyo-ro,

Bundang-gu, Seongnam-si, Kyonggi-do, 13488,

Republic of Korea

Tel.: +82-31-881-7101, Fax: +82-31-881-7069

e-mail: schahm@cha.ac.kr and can lead to sensory, psychological, and motor disorders $^{[1]}$. More than half of the stroke patients suffer from permanent impairment and manifest various symptoms ${ }^{[2]}$. Reduced function of the upper extremity is the most common impairment in patients of acute stroke $^{[3]}$. Moreover, approximately $55 \%-75 \%$ of the patients with stroke show a decrease in activities of daily living due to the residual dysfunction and physical disabilities ${ }^{[4]}$. These deficits affect their quality of life ${ }^{[5]}$.

Virtual reality can be used as a rehabilitation tool, and virtual reality-based therapeutic exercise (VRTE) is 
used as a rehabilitation therapy to improve the physical function in patients with stroke ${ }^{[6,7]}$. An exercise program using virtual reality (VR) is a method of performing a given task by looking directly at the screen, which is enjoyableand effective in improving the physical function $^{[7]}$. Previous studies have reported VRTE as an effective rehabilitative intervention ${ }^{[6,8]}$. Thus, it can be a highly useful intervention for the rehabilitation of stroke patients.

A previous study reported that a program using VR is more effective than conventional rehabilitation therapy to improve the function of the upper extremity and activities of daily living ${ }^{[6]}$. VRTE has positive effects on the functional recovery of the upper and lower extremities, and balance ability for stroke patients has been well documented in the previous studies ${ }^{[9-13]}$. In addition, VRTE using Nintendo Wii has been reported as an effective VRTE intervention for neurological deficits $^{[9,10,12]}$.

Several clinical studies have reported the use of VRTE for improvement of the upper extremity function in stroke patients; these studies involved patients of stroke in the sub-acute or chronic phase ${ }^{[6,8,14]}$. Effects of early use of VRTE for the improvement of upper extremity function and activities of daily living in patients with acute stroke have not yet been investigated. Hence, the aim of this study was to investigate the feasibility of early use of VRTE to improve the function of the upper extremity and activities of daily living in patients with stroke. We compared the effects of VRTE and of occupational therapy (OT) on the upper extremity function and activities of daily living in patients with acute stroke.

\section{Materials and Method}

Study Design: This study was a single-blinded, randomized, controlled trial. It was approved by the Gachon University Institutional Review Board (1044396-201708-HR-136-01). The study was performed in accordance with the protocol, and all participants provided written informed consent prior to enrollment in the study.

Participants: Twenty-four participants were recruited from a medical center located at Incheon. Participants who had (a) suffered a stroke within the last 30 days; (b) upper extremity dysfunction, which the fulfilled criteria $(<50$ in Fugl-Meyer assessment (FMA); over fair grade in manual muscle testing), (c) stable medical condition, and (d) no severe cognitive disorder $(<21$ in mini mental state examination) were included in the study. The exclusion criteria were (a) visual spatial neglect, (b) apraxia, or (c) contracture of the upper extremity.

Experimental Procedures: All participants were randomly divided into either the VRTE or OT groups using the block randomization method ${ }^{[15]}$. Allocation was performed by another employee not related to this study. The assessors were blinded to the intervention allocation and was performed by another therapist who did not perform the intervention. To maintain blinding until the end of the study, participants were asked not to discuss about their groups with the assessors. For allocation concealment, numbered, sealed envelopes with allocation sequences were kept in a room separated from the area where the measurements were performed and were not available to everyone involved in the study until the randomization.

The VRTE group played three different 3 type of sports games: swordplay, table tennis, and canoe in Nintendo Wii Sports Resort. Detailed descriptions can be found at http://wiisportsresort.com/. Participants in the VRTE group can play a rehabilitation game with a remote controller. They performed a variety of movements on the shoulder, elbow, and wrist for VRTE. Those who could not hold the remote controller by degradation of loss of muscle strength or poor grip strength were trained with straps to fasten a remote controller for effective training. In the OT group, OT was performed by occupational therapists for 30 minutes. It consists of strengthening and stretching exercises using full range of motion of the upper extremity, which is a task-oriented therapy. Participants in both groups received general physical therapy including stretching, lower extremity strength, and gait training for 30 minutes. All treatments were performed 5 days per week for 4 weeks ( 20 sessions).

Outcome variables were assessed before the first intervention and after 20 sessions of intervention. Jebsen-Taylor hand function test (JT) and Fugl-Meyer assessment (FMA) were used to assess the upper limb function. Grip strength (GS) and Modified Barthel index (MBI) were used to quantify the grip strength and activities of daily living, respectively. All interventions and assessments were performed by 4 occupational therapists, with a clinical experience of more than 5 years. 
Outcome Measures: JT was used to assess the function of the hand. The reliability of the dominant hand JT score was 0.67.-0.99, while that for the nondominant hand it was $0.60 .0 .92^{[16]}$. We assessed the affectedside and used the Korean version of $\mathrm{JT}^{[17]}$. The maximum score is 105 and minimum is 0 ; a higher score indicates better upper limb function.

FMA was used to test the performance of the upper extremity ${ }^{[18]}$. It has a maximum possible score of 66 and minimum of 0 . The inter-rater reliability of FMA has been reported to be $0.96^{[19]}$.

We used a dynamometer to assess the GS of the hand. The participants were comfortably seated and their affected hand was positioned on their lap. The normal position was the elbow at $90^{\circ}$ with the arm positioned close to the side of the body and neutral position of the wrist. GS was measured 3 times and mean values were used for the analysis ${ }^{[20]}$.

MBI was used to assess the performance in activities of daily living. It comprises 10 activities to be assessed: feeding, grooming, bathing, dressing, toileting, bladder control, bowel control, chair/bed transfer, mobility, and stair climbing. The maximum possible score is 100 , which indicates completely independence in performing activities of daily living. The inter-rater reliability of MBI has been reported to be 0.95 , and the intra-rater reliability is reported to be $0.89^{[21]}$.

Statistical Analysis: SPSS version 18.0 was used for all statistical analyses (SPSS Inc., Chicago, USA). All values are expressed as mean \pm standard deviation (SD). The Wilcoxon-signed rank test was performed to analyze the scores before and after VRTEand OT. The Mann-Whitney $U$ test was used to compare the change in scores between the two groups. A p-value $<0.05$ was considered statistically significant.

\section{Results}

Participant Characteristics: All participants completed the trial. The general characteristics ofthe participants are shown in Table 1, and there were no significant differences between the groups in terms of sex, age, height, weight, stroke type, affected side, duration since onset, and results of the mini mental state examination. There were also no significant differences in the values of the assessment variables between the two groups before the intervention (Table 2).

Table 1. General characteristics between the two groups

\begin{tabular}{|l|c|c|c|}
\hline & VRTE $(\mathbf{n}=\mathbf{1 2})$ & OT (n= 12) & p \\
\hline Sex (Male/female) & \multicolumn{1}{|c|}{0.688} \\
\hline Age (Years) & $7 / 5$ & $6 / 6$ & 0.259 \\
\hline Height (cm) & $71.00 \pm 8.36$ & $165.66 \pm 9.20$ & 0.707 \\
\hline Weight (kg) & $166.66 \pm 8.17$ & $63.50 \pm 10.27$ & 0.840 \\
\hline Stroke type (Ischemic/hemorrhagic) ${ }^{\dagger}$ & $63.54 \pm 9.15$ & $8 / 4$ & 0.660 \\
\hline Affected side (Left/right) ${ }^{\dagger}$ & $9 / 3$ & $8 / 4$ & 0.680 \\
\hline No. of days since onset (Days) & $7 / 5$ & $17.25 \pm 3.13$ & 0.747 \\
\hline MMSE (Score) & $17.66 \pm 3.20$ & $25.33 \pm 2.90$ & 1.000 \\
\hline
\end{tabular}

VRTE, virtual reality based therapeutic exercise; OT, occupational therapy; MMSE, mini mental state examination Values are expressed as mean \pm standard deviation or the number of participants $\dagger$.

Outcome Measures: In the VRTE group, JT ( $p=$ $0.002)$ and FMA $(p=0.002)$ significantly improved after the intervention (Table 2). In the OT group as well, JT $(\mathrm{p}=0.002)$ and FMA $(\mathrm{p}=0.001)$ significantly improved after the intervention (Table 2). Compared with the OT group, the VRTE group did not show significant improvements in JT and FMA (Table 2).
In both, VRTE group and OT groups, GS significantly improved after the intervention $(p=0.002$ for both) (Table 2). The OT group showed significant improvement in GS compared to the VRTE group ( $\mathrm{p}=$ $0.018)$.

In both, VRTE and OT groups, MBI significantly improved after the intervention $(p=0.002$ and $p=0.001$ 
respectively) (Table 2). Compared with the OT group, the VRTE group did not show significant improvements in MBI (Table 2).

Table 2. Comparisons of changes in the outcome variables

\begin{tabular}{|l|l|c|c|c|}
\hline \multicolumn{2}{|c|}{} & VRTE $(\mathbf{n}=\mathbf{1 2})$ & OT $(\mathbf{n}=\mathbf{1 2})$ & p \\
\hline \multirow{5}{*}{ JT (score) } & Pre-test & $27.58 \pm 16.74$ & $27.67 \pm 18.60$ & 0.817 \\
\cline { 2 - 5 } & Post-test & $55.08 \pm 13.56^{*}$ & $55.92 \pm 13.45^{*}$ & 0.544 \\
\cline { 2 - 5 } & Post - Pre & $27.50 \pm 7.13$ & $28.25 \pm 7.20$ & 0.885 \\
\cline { 2 - 5 } & $\mathrm{p}$ & 0.002 & 0.002 & \\
\hline \multirow{5}{*}{ FMA (score) } & Pre-test & $27.50 \pm 5.98$ & $27.75 \pm 3.70$ & 0.464 \\
\cline { 2 - 5 } & Post-test & $47.67 \pm 2.46^{*}$ & $49.75 \pm 3.89^{*}$ & 0.086 \\
\cline { 2 - 5 } & Pre -Post & $20.17 \pm 5.80$ & $22.00 \pm 5.48$ & 0.772 \\
\cline { 2 - 5 } & $\mathrm{p}$ & 0.002 & 0.001 & 0.371 \\
\hline \multirow{5}{*}{ GS (kg) } & Pre-test & $8.19 \pm 3.70$ & $6.24 \pm 3.56$ & 0.525 \\
\cline { 2 - 5 } & Post-test & $13.24 \pm 4.18^{*}$ & $14.76 \pm 3.87^{*}$ & $0.018^{\dagger}$ \\
\cline { 2 - 5 } & Pre -Post & $5.05 \pm 1.32$ & $8.52 \pm 4.07$ & \\
\cline { 2 - 5 } & $\mathrm{p}$ & 0.002 & 0.002 & 0.603 \\
\hline \multirow{5}{*}{ MBI (score) } & Pre-test & $57.83 \pm 12.97$ & $55.17 \pm 14.29$ & 0.885 \\
\cline { 2 - 5 } & Post-test & $31.08 \pm 11.40$ & $33.00 \pm 14.03$ & 0.665 \\
\cline { 2 - 5 } & Pre-Post & 0.002 & 0.001 & \\
\cline { 2 - 5 } & $\mathrm{p}$ & & & \\
\hline
\end{tabular}

VRTE, virtual reality based therapeutic exercise; OT, occupational therapy; JT, Jebsen Taylor hand function test; FMA, Fugl-Meyer assessment (upper limb); GS, grip strength; MBI, modified Barthel index

${ }^{*} \mathrm{p}<0.05$, significant difference within the group; ${ }^{\dagger} \mathrm{p}<0.05$, significant difference between the groups

Values are expressed as mean \pm standard deviation.

\section{Discussion}

This study showed that both, VRTE and OT significantly improved the upper limb function and activities of daily living in patients with acute stroke. However, there were no significant differences between VRTE and OT in the outcome variables. Furthermore, there was a significant improvement in handgrip strength in the OT group compared to that in the VRTE group. VRTE was not superior to OT for rehabilitation of the upper limb function in the acute phase of stroke.

In this study, VRTE significantly improved the upper limb function and activities of daily living in patients with acute stroke; however, these results comparing the effects of virtual reality training and daily OT showed no significant recovery of the motor function. Contrary to the findings of this study, Saposniket al. ${ }^{[12]}$ reported significant differences in grip strength and hand dexterity compared with virtual reality using Wii and recreational therapy. VRTE using Wii was more effective than recreational therapy for improvement in hand function. The reason for the inconsistent results might be because our study used task-repetition based training in the OT group; however, the previous study used recreation therapy as the control group, which showed differences in treatment intensity and form.

Task-oriented training provides opportunities for stroke patients to solve motor problems while performing functional tasks, and is effective in improving the function of the upper extremity and performance of daily activities after stroke ${ }^{[22]}$. According to a previous systematic review, various task-oriented treatments improved the lower and upper extremity functions after stroke ${ }^{[23]}$. Due to the effects of task-oriented training for rehabilitation of stroke paitents, the effect of VRTE seen in this study might fail to be superior to that of taskorientedOT training applied in this study. 
The findings of this study might also be due to the severely degraded upper extremity function after injury. The mean FMA scores of the participants in this study were reported to be around 27, which indicate moderate to severe impairment of the upper limb function ${ }^{[24]}$. Thus, patients with acute stroke had severely reduced hand function and participants in the VRTE group trained with straps to be able to grasp the remote controller during VRTE training. The straps might have restrictedthe movement of their hand and wrist. These results match those of a previous study by Yin et al. ${ }^{[25]}$.

The present study has some limitations. First, the sample size was small. A further study with sample size calculation is necessary to validate the findings of this study. Second, the VRET group used an exercise program provided by Nintendo Wii. To reach a general conclusion, the efficacy of a more suitable VRTE program for rehabilitation of acute stroke patients should be investigated. Third, despite reporting the benefits of VRTE, this study did not investigate mental health such as depression and anxiety. A low level of physical function can lead to depression in patients with stroke ${ }^{[26]}$, and post-stroke depression increases fatigue and incompetence, and decreases the motivation for rehabilitation $^{[27]}$. VRTE might have positive effects such as prevention of depression, and motivate active participation in the rehabilitation programs ${ }^{[8]}$. Future studies considering mental health in the acute stroke phase are needed.

\section{Conclusion}

In conclusion, VRTE was not superior to OT for the improvement in upper limb function and activities of daily living in patients with acute stroke patients. Further studies with a suitable sample size should be performed.

Ethical Approval: It was approved by the Gachon University Institutional Review Board (1044396201708-HR-136-01).

\section{Source of Funding: Self}

\section{Conflict of Interest: Nil}

\section{References}

1. Gresham, G.E.; Phillips, T.F.; Wolf, P.A.; McNamara, P.M.; Kannel, W.B.; Dawber, T.R. Epidemiologic profile of long-term stroke disability: the Framingham study. Arch Phys Med Rehabil 1979, 60, 487-491.https://pubmed.ncbi. nlm.nih.gov/508073/

2. Lawrence, E.S.; Coshall, C.; Dundas, R.; Stewart, J.; Rudd, A.G.; Howard, R.; Wolfe, C.D. Estimates of the prevalence of acute stroke impairments and disability in a multiethnic population. Stroke 2001, 32, 1279-1284, doi:10.1161/01.str.32.6.1279. https://www.ahajournals.org/doi/full/10.1161/01. str.32.6.1279

3. Persson, H.C.; Parziali, M.; Danielsson, A.; Sunnerhagen, K.S. Outcome and upper extremity function within 72 hours after first occasion of stroke in an unselected population at a stroke unit. A part of the SALGOT study. BMC Neurol 2012, 12, 162, doi:10.1186/1471-237712-162.https://bmcneurol.biomedcentral.com/ articles/10.1186/1471-2377-12-162

4. Nakayama, H.; Jørgensen, H.S.; Raaschou, H.O.; Olsen, T.S. Recovery of upper extremity function in stroke patients: the Copenhagen Stroke Study. Arch Phys Med Rehabil 1994, 75, 394-398, doi:10.1016/0003-9993(94)90161-9. https://www.sciencedirect.com/science/article/abs/ pii/0003999394901619?via\%3Dihub

5. Nichols-Larsen, D.S.; Clark, P.C.; Zeringue, A.; Greenspan, A.; Blanton, S. Factors influencing stroke survivors' quality of life during subacute recovery. Stroke 2005, 36, 1480-1484, doi:10.1161/01.STR.0000170706.13595.4f. https://www.ahajournals.org/doi/full/10.1161/01. str.0000170706.13595.4f

6. Laver, K.E.; Lange, B.; George, S.; Deutsch, J.E.; Saposnik, G.; Crotty, M. Virtual reality for stroke rehabilitation. Cochrane database of systematic reviews 2017.https://www.cochrane. org/CD008349/STROKE_virtual-reality-strokerehabilitation

7. Weiss, P.L.; Rand, D.; Katz, N.; Kizony, R. Video capture virtual reality as a flexible and effective rehabilitation tool. J Neuroeng Rehabil 2004, 1, 12, doi:10.1186/1743-0003-1-12. https://jneuroengrehab.biomedcentral.com/ articles/10.1186/1743-0003-1-12

8. Lohse, K.R.; Hilderman, C.G.; Cheung, K.L.; Tatla, S.; Van der Loos, H.F. Virtual reality therapy for adults post-stroke: a systematic review and meta-analysis exploring virtual environments and commercial games in therapy. PLoS One 2014, 9, e93318, doi:10.1371/journal.pone.0093318.https:// journals.plos.org/plosone/article?id=10.1371/ 
journal.pone.0093318

9. Choi, J.H.; Han, E.Y.; Kim, B.R.; Kim, S.M.; Im, S.H.; Lee, S.Y.; Hyun, C.W. Effectiveness of commercial gaming-based virtual reality movement therapy on functional recovery of upper extremity in subacute stroke patients. Ann Rehabil Med 2014, 38, 485-493, doi:10.5535/ arm.2014.38.4.485.https://www.e-arm.org/journal/ view.php?doi=10.5535/arm.2014.38.4.485

10. Celinder, D.; Peoples, H. Stroke patients' experiences with Wii Sports ${ }^{\circledR}$ during inpatient rehabilitation. Scand J Occup Ther 2012, 19, 457463, doi:10.3109/11038128.2012.655307.https:// www.tandfonline.com/doi/abs/10.3109/11038128. 2012.655307.

11. Deutsch, J.E.; Brettler, A.; Smith, C.; Welsh, J.; John, R.; Guarrera-Bowlby, P.; Kafri, M. Nintendo wii sports and wii fit game analysis, validation, and application to stroke rehabilitation. Top Stroke Rehabil 2011, 18, 701-719, doi:10.1310/ tsr1806-701.https://www.tandfonline.com/doi/ abs/10.1310/tsr1806-701

12. Saposnik, G.; Teasell, R.; Mamdani, M.; Hall, J.; McIlroy, W.; Cheung, D.; Thorpe, K.E.; Cohen, L.G.; Bayley, M. Effectiveness of virtual reality using Wii gaming technology in stroke rehabilitation: a pilot randomized clinical trial and proof of principle. Stroke 2010, 41, 14771484, doi:10.1161/strokeaha.110.584979. https://www.ahajournals.org/doi/10.1161/ STROKEAHA.110.584979

13. Deutsch, J.E.; Robbins, D.; Morrison, J.; Bowlby, P.G. Wii-based compared to standard of care balance and mobility rehabilitation for two individuals poststroke. In Proceedings of 2009 virtual rehabilitation international conference; pp. 117-120.https:// ieeexplore.ieee.org/document/5174216

14. Henderson, A.; Korner-Bitensky, N.; Levin, M. Virtual reality in stroke rehabilitation: a systematic review of its effectiveness for upper limb motor recovery. Top Stroke Rehabil 2007, 14, 52-61, doi:10.1310/tsr1402-52.https://www.tandfonline. com/doi/abs/10.1310/tsr1402-52

15. Rosenberger, W.F.; Lachin, J.M. Randomization in clinical trials: theory and practice; John Wiley \& Sons: 2015.https://onlinelibrary.wiley.com/doi/ book/10.1002/0471722103

16. Jebsen, R.H.; Taylor, N.; Trieschmann, R.B.;
Medico-legal Update, April-June 2021, Vol. 21, No. 2

681

Trotter, M.J.; Howard, L.A. An objective and standardized test of hand function. Arch Phys Med Rehabil 1969, 50, 311-319.https://europepmc.org/ article/MED/5788487

17. Kim, J.H.; Kim, I.S.; Han, T.R. New Scoring System for Jebsen Hand Function Test. Journal of the Korean Academy of Rehabilitation Medicine 2007, 31, 623-629.https://www.e-arm.org/journal/ view.php?number $=1425$

18. Fugl-Meyer, A.R.; Jääskö, L.; Leyman, I.; Olsson, S.; Steglind, S. The post-stroke hemiplegic patient. 1. a method for evaluation of physical performance. Scand J Rehabil Med 1975, 7, 13-31.https:// pubmed.ncbi.nlm.nih.gov/1135616/

19. Sanford, J.; Moreland, J.; Swanson, L.R.; Stratford, P.W.; Gowland, C. Reliability of the Fugl-Meyer assessment for testing motor performance in patients following stroke. Phys Ther 1993, 73, 447454, doi:10.1093/ptj/73.7.447.https://academic. oup.com/ptj/article-abstract/73/7/447/2729150

20. Sunderland, A.; Tinson, D.; Bradley, L.; Hewer, R.L. Arm function after stroke. An evaluation of grip strength as a measure of recovery and a prognostic indicator. J Neurol Neurosurg Psychiatry 1989, 52, 1267-1272, doi:10.1136/jnnp.52.11.1267.https:// jnnp.bmj.com/lookup/pmidlookup?view=long \& pmid $=2592969$

21. Granger, C.V.; Albrecht, G.L.; Hamilton, B.B. Outcome of comprehensive medical rehabilitation: measurement by PULSES profile and the Barthel Index. Arch Phys Med Rehabil 1979, 60, 145-154. https://pubmed.ncbi.nlm.nih.gov/157729/

22. Perry, S.B. Stroke rehabilitation: guidelines for exercise and training to optimize motor skill. LWW: 2004.https://journals.lww.com/jnpt/Pages/ articleviewer.aspx?year $=2004 \&$ issue $=06000 \&$ article $=00009 \&$ type $=$ Fulltext

23. Rensink, M.; Schuurmans, M.; Lindeman, E.; Hafsteinsdóttir, T. Task-oriented training in rehabilitation after stroke: systematic review. J Adv Nurs 2009, 65, 737-754, doi:10.1111/j.13652648.2008.04925.x.https://onlinelibrary.wiley. com/doi/full/10.1111/j.1365-2648.2008.04925.x

24. Faria-Fortini, I.; Michaelsen, S.M.; Cassiano, J.G.; Teixeira-Salmela, L.F. Upper extremity function in stroke subjects: relationships between the international classification of functioning, disability, and health domains. J Hand Ther 
2011, 24, 257-264; quiz 265, doi:10.1016/j. jht.2011.01.002.https://www.sciencedirect.com/ science/article/pii/S0894113011000044

25. Yin, C.W.; Sien, N.Y.; Ying, L.A.; Chung, S.F.; Tan May Leng, D. Virtual reality for upper extremity rehabilitation in early stroke: a pilot randomized controlled trial. Clin Rehabil 2014, 28, 1107-1114, doi:10.1177/0269215514532851.https://journals. sagepub.com/doi/abs/10.1177/0269215514532851

26. Sinyor, D.; Amato, P.; Kaloupek, D.G.; Becker, R.; Goldenberg, M.; Coopersmith, H. Post-stroke depression: relationships to functional impairment, coping strategies, and rehabilitation outcome. Stroke 1986, 17, 1102-1107, doi:10.1161/01. str.17.6.1102.https://www.ahajournals.org/ doi/10.1161/01.STR.17.6.1102

27. Schubert, D.S.; Taylor, C.; Lee, S.; Mentari, A.; Tamaklo, W. Physical consequences of depression in the stroke patient. Gen Hosp Psychiatry 1992, 14, 69-76, doi:10.1016/0163-8343(92)90028-9. https://www.sciencedirect.com/science/article/abs/ pii/0163834392900289 